\title{
Feasibility of maintaining near-normal glucose control in pregnant women with type 1 diabetes during COVID-19 lockdown
}

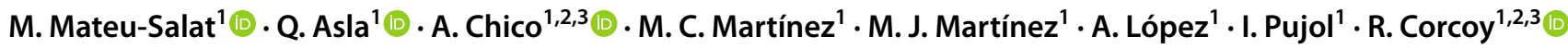

Received: 23 January 2022 / Accepted: 31 January 2022 / Published online: 1 March 2022

c) Springer-Verlag Italia S.r.l., part of Springer Nature 2022

Keywords Type 1 diabetes mellitus · Pregnancy · Times in range · COVID19 · Lockdown

The SARS-CoV-2 pandemic is causing a worldwide social and health crisis. The initial epidemiological situation in Spain compelled the government to set an "alarm state" with institution of a lockdown that limited the mobility of nonessential workers from March 16th to June 19th, 2020. Many reports on glycemic control of patients with diabetes during lockdown have been published [1], but data on pregnant women with type 1 diabetes has not been reported. The aim of this study was to analyze glucose control during lockdown in this group.

We conducted an observational, retrospective study in women with type 1 diabetes that were pregnant during lockdown. The study was approved by the Institutional Review Board and participants gave written informed consent to participate. Inclusion criteria were as follows: type 1 diabetes, follow-up at the Department of Endocrinology and Nutrition at Hospital de la Santa Creu i Sant Pau, last menstrual period before March $9^{\text {th }}$, and use of continuous glucose monitoring (CGM), either real-time (rtCGM) or intermittently scanned (isCGM) since before March 9th. Data sources: electronic health record and Libreview ${ }^{\circledR}$ and Carelink ${ }^{\circledR}$ platforms. Glucose monitoring data were collected from March 9th to June 19th or to last day of pregnancy if earlier. Glucose target values were defined as $63-140 \mathrm{mg} / \mathrm{dL}(3.5-7.8 \mathrm{mmol} / \mathrm{L})$ and targets of time above (TAR), in (TIR), and below range

This article belongs to the topical collection Pregnancy and Diabetes, managed by Antonio Secchi and Marina Scavini.

R. Corcoy

rcorcoy@santpau.cat

1 Department of Endocrinology and Nutrition, Hospital de La Santa Creu I Sant Pau, C/Sant Quintí 89, 08042 Barcelona, Spain

2 CIBER-BBN, Instituto de Salud Carlos III, Madrid, Spain

3 Universitat Autònoma de Barcelona, Barcelona, Spain
(TBR) as $<25 \%,>70 \%$, and $<4 \%$ respectively after Battelino et al [2]. We did not prespecify imputations for missing data. Descriptive statistical analysis was performed with StataMP v14. Variables are expressed as percentages or median values (interquartile range).

Twenty women with type 1 diabetes were pregnant during lockdown and 14 of them fulfilled inclusion criteria (4 of them became pregnant after lockdown initiation, and 2 did not use rtCGM/isCGM or initiated it later).

Average age at the beginning of pregnancy was 36 years (33-39) and diabetes duration 20 years (17-22). Four (29\%) women were previous smokers, five $(36 \%)$ had retinopathy and one had diabetic nephropathy (microalbuminuria). The average prepregnancy body mass index was $23.8 \mathrm{~kg} / \mathrm{m}$ [2] (21-28). Eight (57\%) patients used multiple injection therapy and isCGM, and six (43\%) used sensor-augmented pump therapy (Medtronic 640G®); all women used insulin analogs.

Most patients had specific pregestational follow-up $(n=12 ; 86 \%)$. All pregnancies were singleton, and 3 women ( $21 \%$ ) had undergone an in vitro fertilization process. Average gestational age at the start of lockdown was 13.1 weeks (10-23.1). One woman had a miscarriage. During lockdown, patients had 1.5 (1-2) in-person endocrinological appointments in addition to 5 (3-6) remote asynchronous consultations.

Glucose data per week is displayed in Fig. 1. All participants had information for all included pregnancy weeks. In week 0, average time of sensor use was $99.5 \%$ (95-100), mean blood glucose was $108.5 \mathrm{mg} / \mathrm{dL}$ (99-130) [6.02 mmol/L, (5.5-7.2)]) and TBR, TIR and TAR were respectively $5 \%(4-6 \%), 71.5 \%(59-79 \%)$, and $18.5 \%$ $(8-36 \%)$. The number of women achieving the corresponding target values was 3, 8, and 9. During lockdown, average values were $98.9 \%$ (96.1-100) for time of sensor use, $105 \mathrm{mg} / \mathrm{dL}(102.5-118)$ [5.83 mmol/L, (5.7-6.6)] 


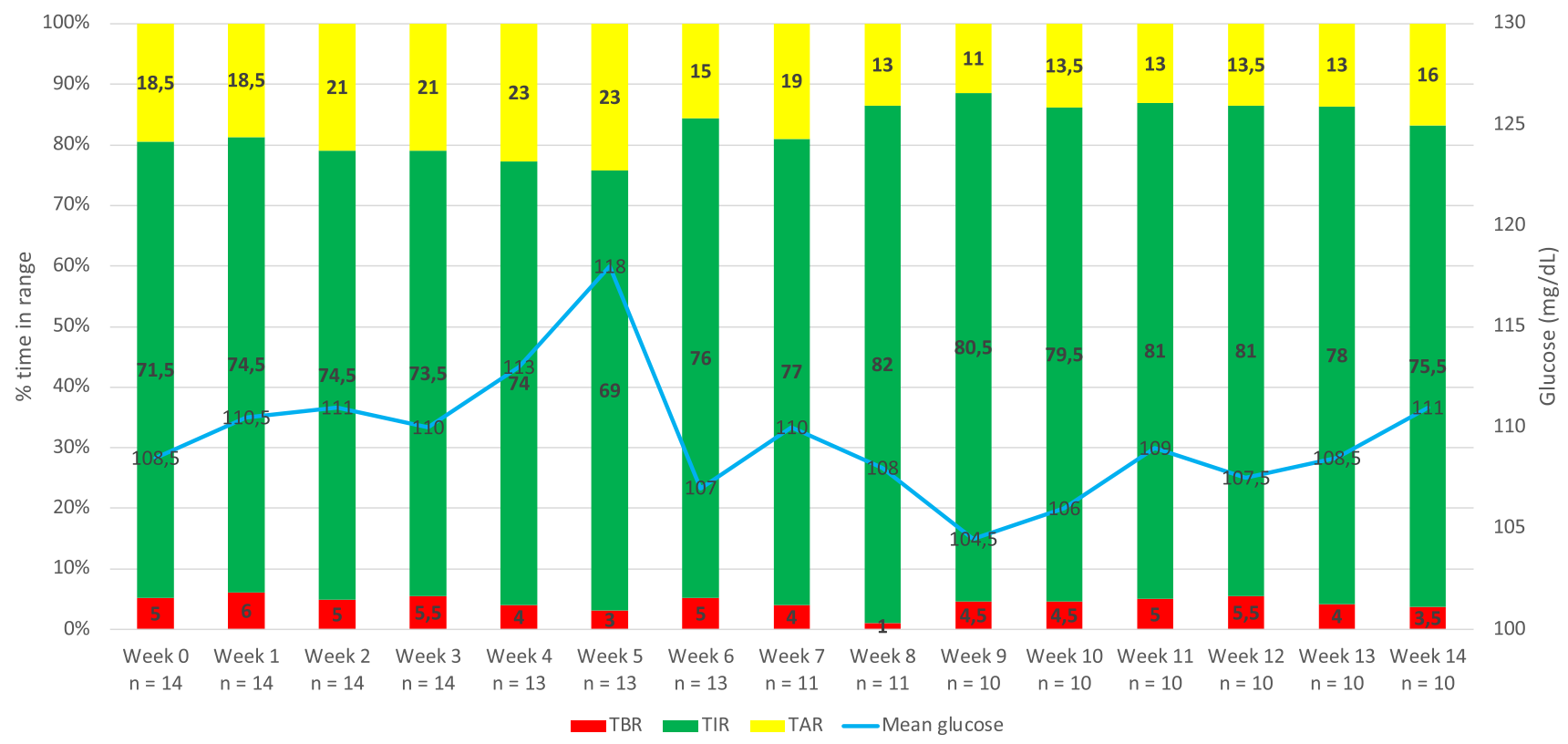

Fig. 1 Glucometrics for pregnant women with type 1 diabetes before and during lockdown in the first wave of COVID19 pandemics

for mean blood glucose, $4 \%(3-10 \%)$ for TBR, $74.25 \%$ (68-80.5\%) for TIR, and $19.5 \%$ (8-28\%) for TAR. Weekto-week glucometrics did not display major modifications and were similar to or nominally better than those of week 0 . According to average values, 5 (36\%) women were in target for TBR during the lockdown period, 9 (64\%) for TIR and 10 (71\%) for TAR. The global picture was similar for women that at week 0 were above or below the median gestational age of 13.1 weeks (data not shown). There were no episodes of severe hypoglycemia (SH) or diabetic ketoacidosis.

Glycemic control in pregnant women with diabetes is a priority in times where resources are limited, such as during a pandemic. In this line, the $\mathrm{ABCD}$ position statement on risk stratification of adult patients with diabetes during COVID-19 pandemic [3] includes as urgent priority those women who are planning pregnancy in the next 6 months. In this report, overall figures of glucose control were satisfactory except for TBR. The low rate of women achieving the TBR target is not necessarily bad news taking into account that no SH episode was reported and that according to a recent report of the CONCEPTT trial [4] achieving the TBR target is associated with a higher risk of pre-eclampsia and neonatal hypoglycemia suggesting that the range may include some readings that would actually be "normal". The satisfactory glucose control is remarkable considering the tight targets and varying insulin requirements during pregnancy. We attribute it to the combination of women's commitment, skilled education, availability of technology, and feasibility of remote consultations. The main limitations of this report are the number of included women and considerations for external validity; its main strengths are the longitudinal analysis, the lack of missing data, and high time of sensor use.

In conclusion, in pregnant women with type 1 diabetes mellitus, glucose metrics after rtCGM/isCGM during lockdown were satisfactory and, in relation to week 0 , were similar or even nominally better. Near-normoglycemia in pregnant women with type 1 diabetes is feasible [5], also in pandemic times.

Funding This research received no specific grant from any funding agency in the public, commercial or not-for-profit sectors.

\section{Declarations}

Conflict of interest M.M.S. has received funds from Novo Nordisk for speaker engagements. A.C. has received funds from Abbott, Lilly, Novo Nordisk, Sanofi, Menarini, Roche Diagnostics, Medtronic, Diabeloop, and Novalab as a consultant for speaker engagements or travel grants. R.C. has received funds from Abbott, Ascensia, Lilly, Medtronic, Menarini, Novo Nordisk, and Sanofi as a consultant for speaker engagements or travel grants.

\section{References}

1. Mesa A, Viñals C, Pueyo I et al (2020) The impact of strict COVID-19 lockdown in Spain on glycemic profiles in patients with type 1 Diabetes prone to hypoglycemia using standalone continuous glucose monitoring. Diabetes Res Clin Pract 167:108354. https://doi.org/10.1016/j.diabres.2020.108354 
2. Battelino T, Danne T, Bergenstal RM et al (2019) Clinical Targets for Continuous Glucose Monitoring Data Interpretation: Recommendations From the International Consensus on Time in Range. Diabetes Care 42(8):1593-1603. https://doi.org/10.2337/ DCI19-0028

3. Nagi D, Wilmot E, Owen K et al (2021) ABCD position statement on risk stratification of adult patients with diabetes during COVID-19 pandemic. Br J Diabetes 21(1):123-131. https://doi. org/10.15277/BJD.2021.282

4. Tundidor D, Meek CL, Yamamoto J et al (2021) Continuous Glucose Monitoring Time-in-Range and HbA 1c Targets in Pregnant Women with Type 1 Diabetes. Diabetes Technol Ther. https://doi. org/10.1089/dia.2021.0073
5. Jovanovic L, Peterson CM, Saxena BB, Dawood MY, Saudek CD (1980) Feasibility of maintaining normal glucose profiles in insulin-dependent pregnant diabetic women. Am J Med 68(1):105112. https://doi.org/10.1016/0002-9343(80)90179-5

Publisher's Note Springer Nature remains neutral with regard to jurisdictional claims in published maps and institutional affiliations. 